\title{
Investigating dosimetric effect of rotational setup errors in IMPT planning of synchronous bilateral lung cancer
}

\author{
Suresh Rana1, Yongbin Zhang2, Gary Larson' ${ }^{3}$, Carlos Vargas4, Megan Dunn', Yuanshui Zheng1 \\ ${ }^{I}$ Department of Medical Physics, ProCure Proton Therapy Center, Oklahoma City, Oklahoma, USA \\ ${ }^{2}$ Department of Medical Physics, Scripps Proton Therapy Center, San Diego, California, USA \\ ${ }^{3}$ Department of Radiation Oncology, ProCure Proton Therapy Center, Oklahoma City, Oklahoma, USA \\ ${ }^{3}$ Radiation Oncology, Proton Collaborative Group (PCG), Warrenville, Illinois, USA
}

Received September 15, 2015; Revised November 01, 2015; Accepted November 15, 2015; Published Online December 01,2015

\section{Original Article}

\begin{abstract}
Purpose: The purpose of this study is to evaluate the dosimetric effect of rotational setup errors on the synchronous bi-lateral lung cancer plans generated by the intensity modulated proton therapy (IMPT) technique. Methods: The original IMPT plans were generated in for the left planning target volume (PTV) and right PTV of the left lung and right lung, respectively. Each plan was generated using two beams (lateral and posterior-anterior) with an isocenter placed at the center of the corresponding PTV. The IMPT plans were optimized for a total dose of $74 \mathrm{~Gy}[\mathrm{RBE}]$ prescribed to each PTV with 2 Gy(RBE) per fraction. Original plans were recalculated by introducing simulated rotational errors. For each PTV, 18 rotational plans $\left( \pm 1^{\circ}, \pm 2^{0}\right.$, and $\pm 3^{0}$ ) for each of the yaw, roll, and pitch rotations were generated. Results: Rotational errors caused the reduction in the clinical target volume (CTV) and PTV coverage in new rotational IMPT plans when compared to the original IMPT lung plans. The CTV D99 was reduced by up to $13.3 \%, 9.1 \%$, and $5.9 \%$ for the yaw $\left(+3^{0}\right)$, roll $\left(-3^{0}\right)$, and pitch $\left(+3^{0}\right)$, respectively. The PTV D95 was reduced by up to $8.7 \%, 7.3 \%$, and $4.6 \%$ for the yaw $\left(+3^{\circ}\right)$, roll $\left(-3^{0}\right)$, and pitch $\left(+3^{0}\right)$, respectively. The PTV V100 showed the highest deviation with a reduction of dose coverage by up to $40.1 \%, 31.8 \%$, and $33.9 \%$ for the yaw $\left(-3^{0}\right)$, roll $\left(-3^{0}\right)$, and pitch $\left(+3^{0}\right)$ respectively. Conclusion: The rotational setup errors with magnitude of $\geq 2^{0}$ can produce a significant loss of dose coverage to the target volume in the IMPT of a synchronous bi-lateral lung cancer. The yaw had the most severe impact on the dosimetric results when compared to other two rotational errors (roll and pitch).
\end{abstract}

Keywords: Proton Therapy; Rotational Setup Error; IMPT; Bilateral Lung Cancer

\section{Introduction}

Proton therapy for the cancer treatment continues to receive wider attention in the radiation oncology and medical physics communities. ${ }^{1}$ The physical properties of protons such as the finite range and sharp distal off behind the Bragg peak allow the deposition of most of the dose within the tumor volume and minimize the doses to the normal tissues and critical structures. ${ }^{1,2}$ Several investigators have investigated the use of proton therapy for various sites such as the prostate cancer ${ }^{3-4}$, lung cancer ${ }^{5-8}$, brain cancer $^{9-10}$, and breast cancer ${ }^{11-12}$. Among different types of cancer, the lung cancer continues to be the leading killer $^{13}$, and various external beam radiation therapy techniques such as proton therapy, 3-dimensional conformal radiation therapy (3CDRT), intensity modulated X-ray therapy (IMXT), and volumetric modulated arc therapy (VMAT) are currently used for the lung cancer treatment.
Earlier treatment planning studies on the lung cancer reported the dosimetric advantages of the proton therapy over the photon therapy (such as 3DCRT, IMXT, etc.). ${ }^{6,14,15,16}$ The study by Zhang et al. ${ }^{17}$, however, showed that the IMXT could provide better dosimetric results than the proton therapy, which used the passive scattering technology. The majority of the previous lung cancer studies included a single lesion, and a very few studies $8,18,19,20$ have investigated the use of radiotherapy for a synchronous bi-lateral lung cancer. The occurrence of a synchronous bi-lateral lung cancer is very rare compared to a single lesion lung cancer. Only two studies $^{8,19}$ have reported the use of proton therapy planning of the synchronous bilateral lung cancer. Rana et al. ${ }^{8}$ showed that the proton therapy provides dosimetric advantages over both the IMXT and VMAT for a synchronous bi-lateral lung cancer. Shi et al. ${ }^{19}$ also reported the dosimetric results using the proton therapy were better than using the IMXT. One of the differences between these two studies was that Rana et 
al. ${ }^{8}$ used the uniform scanning proton therapy (USPT), whereas Shi et al..$^{19}$ used the double scattering proton therapy (DSPT). Although both the studies ${ }^{8,19}$ showed the dosimetric benefits of the proton therapy over the photon therapy, the dosimetric effect of the rotational setup errors in the proton therapy of the synchronous bi-lateral lung cancers was not evaluated.

More recently, proton therapy community is showing a great interest in using the pencil beam scanning (PBS), which is considered as a more advanced technology compared to both the USPT and DSPT. ${ }^{2,20}$ The PBS technique can generate treatment plans with conformal distal and proximal target coverage. However, the PBS delivery can be very sensitive to the motion and setup uncertainties. ${ }^{21}$ The interplay effect between the lung tumor motion and scanned pencil beams is also major concern. The combination of setup errors and interplay effect could result in the target underdosage or normal tissue overdosage. ${ }^{21}$

Although the PBS planning for the lung cancer treatment has been addressed in the past ${ }^{21}$, the dosimetric impact of rotational setup errors on the PBS dose distributions of a bilateral lung cancer is yet to be investigated, especially for the intensity modulated proton therapy (IMPT) planning with multi-field optimization technique (hereafter referred as IMPT).The IMPT planning is typically done by optimizing all fields together to produce uniform dose coverage to the target volume. The study on the dosimetric effect of rotational setup uncertainties is essential in order to ensure the acceptable dose coverage during the lung cancer treatment. The purpose of this study is to evaluate the dosimetric effect of rotational setup errors on the synchronous bi-lateral lung cancer plans generated by the IMPT technique.

\section{Methods and Materials}

\section{Patient selection and simulation}

The computed tomography (CT) dataset of a synchronous bi-lateral lung cancer case (tumor motion less than $1 \mathrm{~cm}$ ) was selected for this study. The patient has consented to participation in the Proton Collaborative Group (PCG) REG001-09, WIRB Protocol \# 20091082. The patient had undergone 4-dimensional (4D) CT simulation in a head first supine position with the support of immobilization devices such as wing board, knee roll, and Vac-lok system (CIVCO Medical Solutions, Kalona, Iowa). The CT images were acquired with a slice thickness of $1.25 \mathrm{~mm}$ using a General Electric CT Scanner.

\section{Contouring}

Since the patient had the synchronous bi-lateral lung cancer, the radiation oncologist delineated the internal gross tumor volume (IGTV) in the left and right lungs (left IGTV $=91.69$ $\mathrm{cc}$ and right IGTV $=35.75 \mathrm{cc}$ ). The clinical target volume (CTV) was generated by a $7 \mathrm{~mm}$ uniform expansion around the IGTV. The planning target volume (PTV) was generated by a $2.5 \mathrm{~mm}$ uniform expansion around the CTV. Normal lung tissue, heart, esophagus, and spinal cord were contoured too.

\section{Treatment planning}

The CT dataset of a synchronous bi-lateral lung cancer case was retrieved from the clinical database of our institution, and imported into the $\mathrm{XiO}$ treatment planning system (version 5.00., CMS Inc, Elekta, St. Louis, MO) for the IMPT planning. The control IMPT plans were created in the XiO TPS, which used the beam commissioning data measured on an IBA Cyclotron (IBA, Louvain-la-Neuve, Belgium). The average CT dataset (obtained from the 4DCT scan) was used for proton planning. The left and right IMPT plans were generated for the left PTV and right PTV, respectively by using a 2-field (lateral and posterior-anterior) technique as shown in the Figure 1. Each plan (left and right lung) had its own isocenter, which was placed at the center of the corresponding PTV. The IMPT plans (left lung and right lung) were optimized for a total dose of 74 Gy[RBE] prescribed to each PTV (left and right) with $2 \mathrm{~Gy}(\mathrm{RBE})$ per fraction. In each plan, the layer spacing was set to $8 \mathrm{~mm}$, and the proton dose calculations were performed using a pencil beam algorithm ${ }^{22}$ with a grid size of $3 \mathrm{~mm} \times 3 \mathrm{~mm} \times 3 \mathrm{~mm}$.

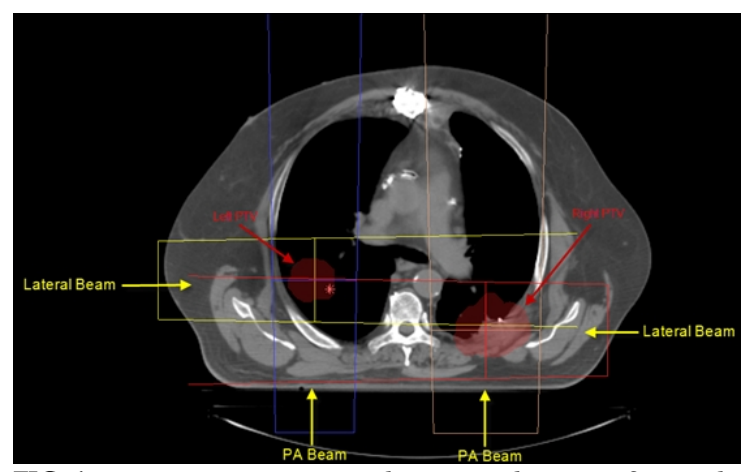

FIG. 1: Beam arrangements in the IMPT planning of a synchronous bi-lateral lung cancer. Each PTV (left and right lung) has its own isocenter.

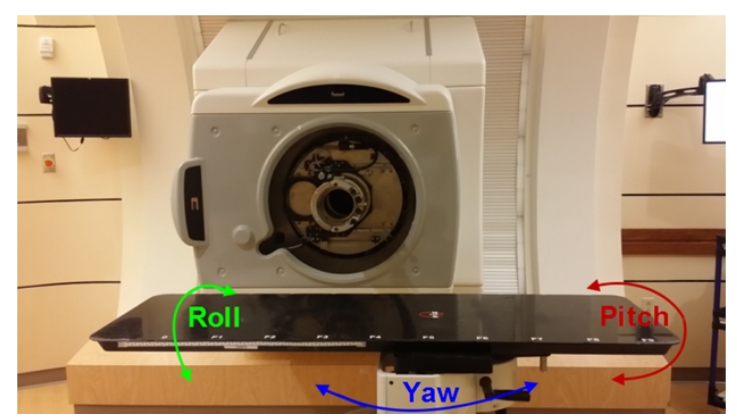

FIG. 2: Simulation of rotational setup errors (yaw, roll, and pitch) by changing couch angles. 
In order to investigate the rotational setup errors, the original plans (or control plans) were recalculated by introducing simulated rotational errors (roll, yaw, and pitch) but keeping the same treatment parameters as for the original left and right lung plans. Specifically, 18 rotational plans for each tumor $\left( \pm 1^{0}, \pm 2^{0}\right.$, and $\left.\pm 3^{0}\right)$ for each of the yaw, roll, and pitch rotations were generated. The yaw, roll, and pitch rotations were simulated by changing one parameter (e.g., yaw) and keeping other two parameters (e.g., roll and pitch) fixed. [Figure 2] The rotational setup errors were assumed to have occurred for each individual fraction.

The CTV was evaluated for the dose at the $99 \%$ of the CTV (D99). The PTV was evaluated for the dose at the $95 \%$ of the PTV (D95) and the relative volume of the PTV receiving at least $100 \%$ and $95 \%$ of the prescription dose (V100 and V95, respectively). For a comparative purpose, the difference in various dosimetric parameters between the control (or original) plan and new simulated plans was calculated. The difference (D) was calculated as:

$$
D(r)=\frac{X(r)-X(c)}{X(c)} \times 100-----(1)
$$

where, $\mathrm{X}=$ dosimetric parameter (e.g. CTV D99, PTV V95, etc.); $\mathrm{X}(\mathrm{r})=$ dosimetric result in a new plan with a simulated rotation ( $r)\left(r= \pm 1^{0}, \pm 2^{0}\right.$, and $\pm 3^{0}$ for each of the yaw, roll, and pitch); and $\mathrm{X}$ (c) = dosimetric result in the control (c) treatment plan.

\section{Results}

Figure 3 illustrates the difference (see equation 1) in the dosimetric result for a given parameter between the original/control plan and rotational plans for various yaw $\left( \pm 1^{0}, \pm 2^{0}\right.$, and $\left.\pm 3^{0}\right)$, roll $\left( \pm 1^{0}, \pm 2^{0}\right.$, and $\left.\pm 3^{0}\right)$, and pitch $\left( \pm 1^{0}\right.$, $\pm 2^{0}$, and $\pm 3^{0}$ ) rotations.

\section{CTV}

The rotational errors resulted CTV coverage loss as the differences in the CTV D99 were up to $-13.3 \%,-5.6 \%$, and $-5.9 \%$ for the yaw $\left(+3^{0}\right)$, roll $\left(+3^{0}\right)$, and pitch $\left(-3^{0}\right)$, respectively, in the left lung, and up to $-10.3 \%,-9.1 \%$, and $-5.6 \%$ for the yaw $\left(-3^{0}\right)$, roll $\left(-3^{0}\right)$, and pitch $\left(+3^{0}\right)$, respectively, in the right lung.

\section{PTV}

For the PTV D95, the differences were up to $-8.7 \%,-4.1 \%$, and $-2.8 \%$ for the yaw $\left(+3^{0}\right)$, roll $\left(+3^{0}\right)$, and pitch $\left(-3^{0}\right)$, respectively, in the left lung, and up to $-8.4 \%,-7.3 \%$, and $-4.6 \%$ for the yaw $\left(-3^{0}\right)$, roll $\left(-3^{0}\right)$, and pitch $\left(+3^{0}\right)$, respectively, in the right lung. For the PTV V95, the differences were up to $-8.0 \%,-2.9 \%$, and $-1.7 \%$ for the yaw $\left(+3^{0}\right)$, roll $\left(+3^{0}\right)$, and pitch $\left(-3^{0}\right)$, respectively, in the left lung, and up to $-10.8 \%,-9.3 \%$, and $-2.9 \%$ for the yaw $\left(-3^{0}\right)$, roll $\left(-3^{0}\right)$, and pitch $\left(+3^{0}\right)$, respectively, in the right lung. The highest difference was obtained for the PTV V100 as the differences were up to $-23.1 \%,-14.3 \%$, and $-11.1 \%$ for the yaw $\left(+3^{0}\right)$, roll $\left(+3^{0}\right)$, and pitch $\left(-3^{0}\right)$, respectively, in the left lung, and up to $-40.1 \%,-31.8 \%$, and $-33.9 \%$ for the yaw $\left(-3^{0}\right)$, roll $\left(-3^{0}\right)$, and pitch $\left(+3^{0}\right)$, respectively, in the right lung.

\section{Discussion}

In this paper, the dosimetric effect of rotational errors (yaw, roll, and pitch) on the IMPT planning of the synchronous bi-lateral lung cancer was investigated. The results from our study demonstrated that the rotational errors can produce a significant loss of dose coverage in the IMPT lung cancer treatment. The yaw had the most severe impact on the dosimetric results when compared to other two rotational errors (roll and pitch). For $\pm 3^{0}$ yaw error, the difference was found to be more than $-10 \%$ (up to $-13.3 \%$ for the CTV D99, $-10.83 \%$ for the PTV V95, and $-40.1 \%$ for the PTV V100). Even for a smaller degree of rotational setup error, the dosimetric impact could be significant. For the smallest degree of rotation $\left( \pm 1^{0}\right)$ evaluated in this study, the differences in the CTV D99 were up to $-7.63 \%$ for the yaw, up to $-3.3 \%$ for the roll, and up to $-5.6 \%$ for the pitch rotations. These results demonstrate that the loss of dose coverage to the CTV and PTV could be clinically unacceptable if the rotational errors are not applied during the patient treatment setup.

Another interesting observation from this study was that the differences in the dosimetric results were not consistent despite the same magnitude of rotation. For example, the CTV D99 of the left lung had the differences of $-3.2 \%$ for $-3^{0}$ yaw and $-13.3 \%$ for $+3^{0}$ yaw. Similar discrepancy was observed for the right lung as the CTV D99 had the differences of $-10.3 \%$ for $-3^{0}$ yaw and $-3.6 \%$ for $+3^{0}$ yaw. The PTV D95 of the right lung had the differences of $-7.3 \%$ and $-0.8 \%$ at $-3^{0}$ and $+3^{0}$ roll, respectively. These results suggest that the difference in the dose coverage may vary even if the magnitude of the rotational error is the same (e.g., $3^{0}$ of yaw). This may be due to the change in range of proton beam as a result of rotational errors, and proton beams are sensitive to the changes to the tissue density along the beam path and tissue inhomogeneities

We evaluated the dose coverage to the CTV and PTV only. The dosimetric effects of the rotational setup errors on the OARs were not considered because, the beam entrance through critical structures was avoided, and the critical structures were not in the proximity of the target volume. However, the impact of rotational errors on the OARs may be essential when the critical structure such as the spinal cord is abutting the target volume.

Currently, our institution (ProCure Proton Therapy Center $\mathrm{OKC}$ ) uses the USPT technique to treat the lung cancer with 
tumor motion less than $1 \mathrm{~cm}$. However, the lung tumor motion would be even more critical for the PBS. The review article by Wink et al. ${ }^{20}$ reported that the MD Anderson Cancer Center has started using PBS for the treatment of non-small-cell lung cancer with tumor motion below $5 \mathrm{~mm}$. The use of PBS for the lung cancer treatment is yet to be fully explored and need further studies because of the uncertainties involved between the tumor motion and scanned pencil beams. For the lung cancer treatment using the USPT technique, the rotational setup tolerance at our institution is $\pm 2^{0}$ for the yaw, roll, and pitch rotations. Based on the results presented in this article, the rotational setup tolerance of $\pm 2^{0}$ may need to be re-evaluated for the lung cancer treatment using PBS technology.
One of the limitations of our study is the limited number of bilateral lung cases. Due to unavailability of additional bi-lateral lung cancer cases in our current database, we were unable to investigate the impact of rotational errors on the tumors of different shape and size. The interplay effect was also not investigated. Future studies need to address the impact of setup errors and interplay effect on the PBS planning of highly irregular lung tumors by including a large cohort of bilateral lung cancer patients.

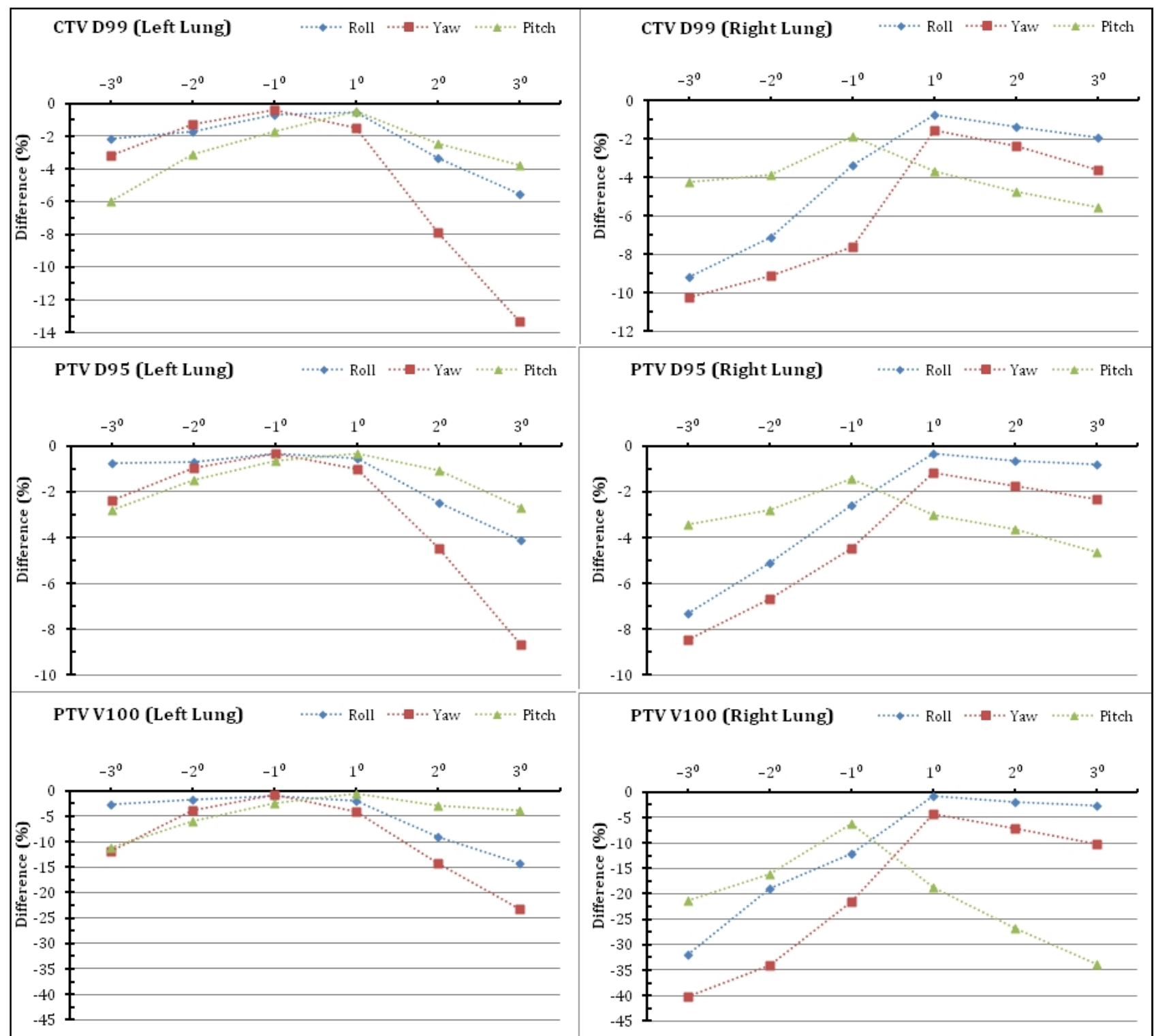

FIG. 3: Difference between the control plans and new rotational plans for various yaw $\left( \pm 1^{0}, \pm 2^{0}\right.$, and $\left.\pm 3^{0}\right)$, roll $\left( \pm 1^{0}, \pm 2^{0}\right.$, and $\left.\pm 3^{0}\right)$, and pitch $\left( \pm 1^{0}, \pm 2^{0}\right.$, and $\left.\pm 3^{0}\right)$ rotations. The results are presented for the left lung and right lung plans (synchronous bi-lateral lung cancer case). 


\section{Conclusion}

The rotational setup errors with magnitude of $\geq 2^{0}$ can produce a significant loss of dose coverage to the target volume in the IMPT of a synchronous bi-lateral lung cancer. The yaw had the most severe impact on the dosimetric results when compared to other two rotational errors (roll and pitch).

\section{Conflict of interest}

The authors declare that they have no conflicts of interest. The authors alone are responsible for the content and writing of the paper.

\section{References}

1. Arjomandy B. Proton therapy advancement. Jour Proton Ther. 2015;1:115.

2. Wilson R. Radiological use of fast protons. Radiology. 1946;47:487-91.

3. Vargas C, Fryer A, Mahajan C, et al. Dose-volume comparison of proton therapy and intensity-modulated radiotherapy for prostate cancer. Int J Radiat Oncol Biol Phys. 2008;70: 744-51.

4. Rana S, Cheng C, Zheng Y, et al. Dosimetric study of uniform scanning proton therapy planning for prostate cancer patients with a metal hip prosthesis, and comparison with volumetricmodulated arc therapy. J Appl Clin Med Phys. 2014;15:4611.

5. Bush DA, Slater JD, Shin BB, et al. Hypofractionated proton beam radiotherapy for stage I lung cancer. Chest. 2004;126:1198-203.

6. Chang JY, Zhang X, Wang X, et al. Significant reduction of normal tissue dose by proton radiotherapy compared with three-dimensional conformal or intensity-modulated radiation therapy in Stage I or Stage III non-small-cell lung cancer. Int J Radiat Oncol Biol Phys. 2006;65: 1087-96.

7. Nakayama H, Sugahara S, Tokita M, et al. Proton beam therapy for patients with medically inoperable stage I non-small-cell lung cancer at the university of tsukuba. Int J Radiat Oncol Biol Phys. 2010;78:467-71.

8. Rana S, Pokharel S, Zheng Y, Zhao L, Risalvato D, Vargas C, Cersonsky N. Treatment planning study comparing proton therapy, RapidArc and IMRT for a synchronous bilateral lung cancer case. Int J Cancer Ther Oncol. 2014;2:020216.

9. Moteabbed M, Yock TI, Paganetti H. The risk of radiation-induced second cancers in the high to medium dose region: a comparison between passive and scanned proton therapy, IMRT and VMAT for pediatric patients with brain tumors. Phys Med Biol. 2014;59:2883-99.

10. Yock T, Schneider R, Friedmann A, Adams J, Fullerton B, Tarbell N. Proton radiotherapy for orbital rhabdomyosarcoma: clinical outcome and a dosimetric comparison with photons. Int J Radiat Oncol Biol Phys. 2005;63:116-8.

11. Lin LL, Vennarini S, Dimofte A, Ravanelli D, Shillington K, Batra S, Tochner Z, Both S, Freedman G. Proton beam versus photon beam dose to the heart and left anterior descending artery for left-sided breast cancer. Acta Oncol. 2015;19:1-8.

12. Ares, C., Khan, S., Macartain, A.M. et al, Postoperative proton radiotherapy for localized and locoregional breast cancer: Potential for clinically relevant improvements?. Int J Radiat Oncol Biol Phys. 2010;76:685-97.

13. American Cancer Society. Cancer Facts \& Figures 2013. Atlanta: American Cancer Society; 2013.

14. Nichols RC, Huh SN, Henderson RH, et al. Proton radiation therapy offers reduced normal lung and bone marrow exposure for patients receiving dose-escalated radiation therapy for unresectable stage iii non-small-cell lung cancer: a dosimetric study. Clin Lung Cancer. 2011;12:252-7.

15. Macdonald OK, Kruse JJ, Miller JM, et al. Proton beam radiotherapy versus three-dimensional conformal stereotactic body radiotherapy in primary peripheral, early-stage non-small-cell lung carcinoma: a comparative dosimetric analysis. Int J Radiat Oncol Biol Phys. 2009;75:950-8.

16. Wang C, Nakayama $H$, Sugahara $S$, et al. Comparisons of dose-volume histograms for proton-beam versus 3-D conformal x-ray therapy in patients with stage I non-small cell lung cancer. Strahlenther Onkol. 2009;185:231-4.

17. Zhang X, Li Y, Pan X, et al. Intensity-modulated proton therapy reduces the dose to normal tissue compared with intensity-modulated radiation therapy or passive scattering proton therapy and enables individualized radical radiotherapy for extensive stage IIIB non-small-cell lung cancer: a virtual clinical study. Int J Radiat Oncol Biol Phys. 2010;77:357-66.

18. Sinha B, McGarry RC. Stereotactic body radiotherapy for bilateral primary lung cancers: the Indiana University experience. Int J Radiat Oncol Biol Phys. 2006;66:1120-4.

19. Shi W, Nichols RC, Flampouri S, et al. Proton-based chemoradiation for synchronous bilateral non-small-cell lung cancers: A case report. Thoracic Cancer. 2013;4:198-202. 
20. Rana S, Larson G, Vargas C, Dunn M, Zheng Y. Intensity modulated proton therapy versus uniform scanning proton therapy: Treatment planning study of the prostate cancer in patients with a unilateral metallic hip prosthesis. Jour Proton Ther. 2015; 1:113.198-202.

21. Wink KC, Roelofs E, Solberg T, Lin L, Simone CB 2nd, Jakobi A, Richter C, Lambin P, Troost EG.
Particle therapy for non-small cell lung tumors: where do we stand? A systematic review of the literature. Front Oncol. 2014;4:292.

22. Hong L, Goitein M, Bucciolini M, et al. A pencil beam algorithm for proton dose calculations. Phys Med Biol. 1996;41:1305-30. 